\title{
Observation of preformed electron-hole Cooper pairs in highly excited $\mathrm{ZnO}$
}

\author{
Marijn A. M. Versteegh, ${ }^{1}$ A. J. van Lange, ${ }^{1}$ H. T. C. Stoof, ${ }^{2}$ and Jaap I. Dijkhuis ${ }^{1, *}$ \\ ${ }^{1}$ Debye Institute for Nanomaterials Science, Utrecht University, Princetonplein 1, 3584 CC Utrecht, The Netherlands \\ ${ }^{2}$ Institute for Theoretical Physics, Utrecht University, Leuvenlaan 4, 3584 CE Utrecht, The Netherlands
}

(Received 17 November 2011; published 15 May 2012)

\begin{abstract}
Electrons and holes in a semiconductor form hydrogen-atom-like bound states, called excitons. At high electron-hole densities the attractive Coulomb force becomes screened and excitons can no longer exist. BCS theory predicts that at such high densities cooperative many-body effects can at low temperatures induce a bound state, an electron-hole Cooper pair, comparable to an electron-electron Cooper pair in a superconductor. Here we report the observation of preformed electron-hole Cooper pairs in a semiconductor. By measuring stimulated emission from a dense electron-hole gas in $\mathrm{ZnO}$, we have explored both the crossover from the electron-hole plasma to the preformed Cooper-pair regime, and the crossover from the exciton to the preformed Cooper-pair regime.
\end{abstract}

DOI: 10.1103/PhysRevB.85.195206

PACS number(s): 71.35.Lk, 71.10.Li, 78.45.+h, 78.55.Et

\section{INTRODUCTION}

Superconductivity was successfully explained in 1957 by Bardeen, Cooper, and Schrieffer ${ }^{1}$ as the result of Bose-Einstein condensation of many-body-induced bound states of two electrons, called "Cooper pairs." In principle, Bardeen-CooperSchrieffer (BCS) theory also allows for many-body-induced bound states of other kinds of fermionic particles. For example, in the last decade condensates of Cooper pairs consisting of two fermionic atoms have been observed. ${ }^{2-4}$

In 1964, Keldysh and Kopaev predicted the possibility of Cooper pairs of an electron and a hole. ${ }^{5}$ The difference between electron-hole (e-h) Cooper pairs and excitons is in the binding mechanism. Excitons are bound by the Coulomb attraction between one electron and one hole only. The binding of an e-h Cooper pair is a cooperative many-body effect, where, besides e-h Coulomb attraction, Pauli blocking plays an essential role as in the case of electron-electron (e-e) Cooper pairs in a superconductor. This Pauli blocking occurs to a sufficient degree in highly degenerate e-h gases. A crossover from excitons to e-h Cooper pairs has been predicted for increasing density at low temperatures. ${ }^{6}$ Note that in this paper we reserve the term "exciton" for hydrogen-atom-like e-h pairs that are bound due to the Coulomb attraction only.

A related many-body phenomenon is that of the Mahan exciton. $^{7-12}$ A Mahan exciton results from the many-body interaction between a single localized hole and a highly degenerate electron gas inside a doped semiconductor. e-h Cooper pairs are different from Mahan excitons in the sense that e-h Cooper pairs require both the electron gas and the hole gas to have a Fermi surface. This removes the Fermi-edge singularity from the bound-state problem. In the context of an atomic Fermi mixture the Mahan exciton is known as a (spin) polaron or Fermi polaron. ${ }^{13}$

Conditions of high degeneracy, enabling the formation of e-h Cooper pairs, could for example be met at low temperatures in a narrow-band-gap semiconductor or semimetal where the exciton binding energy exceeds the (positive or negative) band gap. ${ }^{5,14,15}$ In such a system, the e-h BCS state, i.e., a condensate of e-h Cooper pairs, also called an "excitonic insulator," would be the appropriate ground state. Indeed, experimental evidence for the excitonic insulator state has been found in $1 T-\mathrm{T}_{1} \mathrm{Se}_{2},{ }^{16,17}$ in $\mathrm{TmSe}_{0.45} \mathrm{Te}_{0.55},{ }^{18}$ and in $\mathrm{Ta}_{2} \mathrm{NiSe}_{5} .{ }^{19}$
Theoretically, also in a highly excited normal semiconductor e-h Cooper pairs are possible. Here, the e-h BCS state would be an inherently nonequilibrium phenomenon. e-h Cooper pairs in highly excited semiconductors, including the crossover between the excitonic Bose-Einstein condensate (BEC) and the e-h BCS state have been extensively studied theoretically. ${ }^{6,20-27}$ Yet, observations of these phenomena were never reported. Recent observations suggest the formation of an excitonic BEC in a single crystal of the direct semiconductor $\mathrm{CuO}_{2} \cdot{ }^{28}$

Quantum well bilayers, where pairs are formed by electrons and holes in separate quantum wells, are considered as promising systems for detecting e-h Cooper pairs. ${ }^{29-31}$ Experimental evidence for excitonic BEC in such systems has been found, ${ }^{32,33}$ as well as in quantum Hall e-e bilayers in high magnetic fields, ${ }^{34,35}$ but, as far as we know, there are no reports of e-h Cooper pairs in bilayer systems. Graphene bilayers $^{36}$ and topological insulators ${ }^{37,38}$ are also very interesting possibilities.

In semiconductor-based microcavities, condensation is more easily accessible, thanks to the very small mass of the polaritons. Bose-Einstein condensates of exciton polaritons have indeed been observed in such systems, ${ }^{39-41}$ but e-h Cooper pairs still need to be demonstrated.

Not only the condensed superfluid phase, but also the normal phase above the superfluid critical temperature is extremely interesting. There, precursors of the condensed e-h Cooper pairs and of the BEC-BCS crossover could exist. ${ }^{42,43}$ These uncondensed or "preformed" e-h Cooper pairs are especially important, since preformed e-e Cooper pairs seem to be related somehow to high-temperature superconductivity. ${ }^{44,45}$ In atomic systems evidence for such uncondensed Cooper pairs has been found at extremely low temperatures. ${ }^{46}$

Here we present the first experimental observation of preformed electron-hole Cooper pairs in a semiconductor. This is achieved by highly exciting a $\mathrm{ZnO}$ single crystal via threephoton absorption at standard cryogenic temperatures and simply measuring the light emission. In contrast to e-e Cooper pairs, e-h Cooper pairs can recombine, and are therefore subject to spontaneous and stimulated emission of photons. We detected the amplified spontaneous emission (ASE) from preformed e-h Cooper pairs. Our experiments explored the 
crossover from the e-h plasma to the preformed e-h Cooperpair gas for decreasing temperature, and the crossover from the exciton gas to the preformed e-h Cooper-pair gas for increasing e-h density. Experimental results are compared with quantum many-body theoretical calculations of the phase diagram and the gain spectra of the e-h gas in $\mathrm{ZnO}$.

\section{PHASE DIAGRAM}

To explain our observations, we calculated the phase diagram of the e-h gas in $\mathrm{ZnO}$, which is shown in Fig. 1. For moderate densities and for temperatures above the critical temperature $T_{C}$, electrons and holes form an almost ideal gas of excitons (region 1). These excitons undergo Bose-Einstein condensation below $T_{C}$ (region 2). ${ }^{47,48}$ When the e-h density of the BEC is increased, the Coulomb forces become gradually screened, leading to a weaker binding of the excitons. Above the so-called Mott density, the attractive Coulomb force is too weak for excitons to exist. In this high-density, lowtemperature regime BCS theory predicts a condensate of e-h Cooper pairs (region 3). The binding of an e-h Cooper pair is a combined result of weak Coulomb attraction and Pauli blocking in the degenerate electron and hole Fermi gases. The energy level of the pairs does not reside within the band gap, as is the case for excitons, but is located near the e-h Fermi level, which is defined as the energy interval between the electron Fermi level and the hole Fermi level. The condensate of e-h Cooper pairs opens up a gap in the e-h pair energy spectrum.

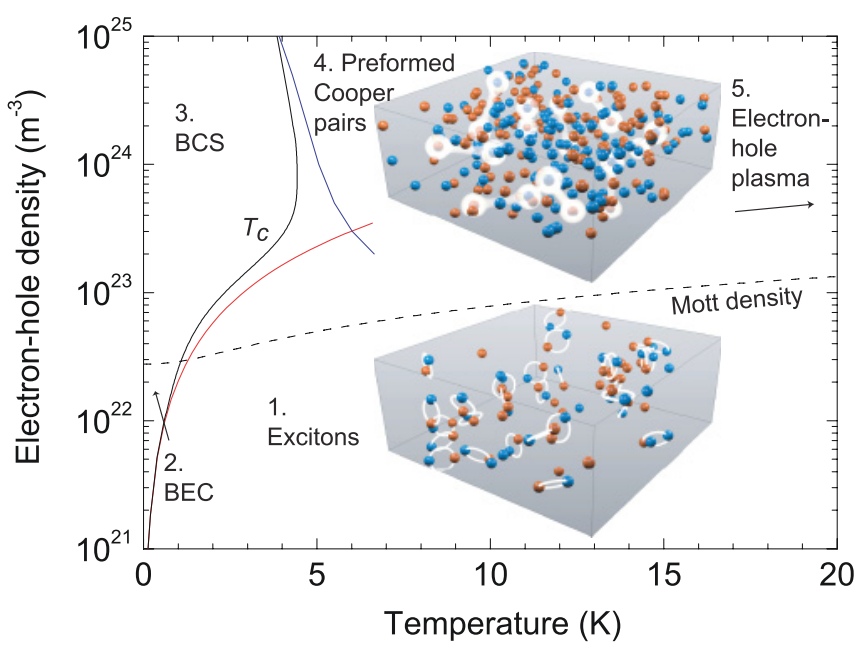

FIG. 1. (Color online) Phase diagram of the electron-hole gas in $\mathrm{ZnO}$. There are five regions: (1) exciton gas, (2) excitonic Bose-Einstein condensate, (3) electron-hole BCS state: a condensate of electron-hole Cooper pairs (also called "nonequilibrium excitonic insulator"), (4) preformed electron-hole Cooper-pair gas, and (5) electron-hole plasma. The blue line is the calculated mean-field critical temperature $T_{C}$ in the BCS regime. The red line is the ideal-gas $T_{C}$ for Bose-Einstein condensation, valid in the BEC regime. Note that the solid black line is the only line of true phase transitions in the phase diagram separating the normal and superfluid phases of the e-h gas. In contrast, the dashed line denoting the Mott density represents only a smooth crossover between different regions in the phase diagram. The insets are visualizations of the electron-hole gas in the exciton regime and in the preformed Cooper-pair regime.
It is interesting to compare the phase diagram of the e-h gas with that of a cold gas of fermionic atoms. While in our case the interaction strength between electrons and holes can be tuned by varying the e-h density, the interaction strength between fermionic atoms can be controlled using a Feshbach resonance. ${ }^{49}$ By sweeping an external magnetic field across a Feshbach resonance, weakly bound diatomic molecules can be created ${ }^{50}$ which are the analog of excitons. Below a critical temperature these molecules form a BEC. ${ }^{51}$ Atomic Cooper pairs can be made by tuning the magnetic field to the other side of the Feshbach resonance. ${ }^{2}$ In particular, the BCS gap $^{3}$ and vortices ${ }^{4}$ have been observed in the atomic BCS superfluid. Recently, it has also been demonstrated that a temperature range exists above the critical temperature, where uncondensed atomic Cooper pairs are present, accompanied by a pseudogap in the energy spectrum. ${ }^{46}$ Pseudogaps and uncondensed Cooper pairs have also been found in high- $T_{C}$ superconductors above the critical temperature. However, in that case the relation between those two is more complicated and less understood than for atomic systems. ${ }^{44,45}$

Returning to our phase diagram, we anticipate that such a regime of uncondensed Cooper pairs also exists for the e-h gas (region 4). In agreement with common practice, we call the uncondensed pairs "preformed electron-hole Cooper pairs," while the term "electron-hole Cooper pair" refers to the condensed state. When the temperature is increased above a crossover temperature $T^{*}>T_{C}$, the preformed e-h Cooper pairs dissociate, resulting in an e-h plasma (region 5). Lowering the density below the Mott density leads to the formation of excitons again.

The Mott density in Fig. 1 was calculated by describing the screened attractive Coulomb interaction between an electron and a hole by the Yukawa potential

$$
V_{s}(r, n, T)=\frac{-e^{2}}{4 \pi \varepsilon_{0} \varepsilon_{r} r} e^{-r / \lambda_{s}(n, T)},
$$

where $r$ is the distance between the electron and the hole, $-e$ is the electron charge, $\varepsilon_{0}$ is the vacuum permittivity, $\varepsilon_{r}$ is the relative dielectric constant of $\mathrm{ZnO}$, and $\lambda_{s}(n, T)$ is the screening length, depending on the e-h density $n$ and the temperature $T$. An excitonic bound state exists in this potential when $\lambda_{s}(n, T)>a_{0}$, where $a_{0}$ is the unscreened exciton Bohr radius. ${ }^{52}$ Therefore, at the Mott density $\lambda_{s}(n, T)=a_{0}$. In $\mathrm{ZnO}$ $a_{0}=4 \pi \hbar^{2} \varepsilon_{0} \varepsilon_{r} /\left(e^{2} m_{r}\right)=1.83 \mathrm{~nm}$, where $m_{r}=0.19 m_{0}$ is the reduced mass of the exciton and $m_{0}$ is the bare electron mass.

The screening length is calculated in the random-phase approximation (RPA) from the ideal-gas chemical potentials of the electron and hole gases, $\mu_{e}(n, T)$ and $\mu_{h}(n, T)$, respectively. For details we refer to Ref. 53. We observe in Fig. 1 that the Mott density rises for increasing temperature, reflecting the reduced screening efficiency by the electrons and holes due to their thermal motion.

For the critical temperature in the BEC regime we used the well-known ideal gas result ${ }^{54}$

$$
T_{C}(n)=\frac{2 \pi \hbar^{2}}{m_{e}+m_{h}}\left(\frac{n}{4 \times 2.612}\right)^{2 / 3},
$$

where $m_{e}=0.28 m_{0}$ and $m_{h}=0.59 m_{0}$ are the effective electron and hole masses in $\mathrm{ZnO}$, respectively. ${ }^{55,56}$ The factor 4, 
multiplying 2.612, is included because of the four possible spin states of the exciton. ${ }^{20}$ The effective interaction between two excitons is at present unknown, but is most likely repulsive, corresponding to a positive scattering length. In that case it would lead to a small upward shift of the critical temperature proportional to $n^{1 / 3}$. This effect is, however, neglected here.

To obtain $T_{C}$ in the BCS regime, we solved the BCS gap equation in mean-field approximation. Details of this calculation are given in the Appendix. The most important result is that the temperatures and densities at which preformed e-h Cooper pairs form in $\mathrm{ZnO}$ seem to be well accessible by experiment. Even reaching the superfluid phase might be quite feasible.

\section{EXPERIMENTAL METHOD}

To explore the physics of the phase diagram, we measured the light emission from a highly excited $\mathrm{ZnO}$ single crystal. The crystal was $500 \mu \mathrm{m}$ thick and oriented in the [0001] direction. $\mathrm{ZnO}$ was chosen for our study for several reasons. First because its strong e-h Coulomb pairing, apparent in the large exciton binding energy of $60 \mathrm{meV}$, leads to a high $T_{C}$. Secondly because its direct band gap results in strong light emission from the crystal. Thirdly because its simple direct band structure prevents the formation of an e-h liquid or e-h droplets. In the indirect semiconductors $\mathrm{Si}$ and $\mathrm{Ge}$, for example, the many-valley structure of the conduction bands, the fourfold degeneracy of the valence bands, and the anisotropy of the corresponding masses lead to the formation of e-h liquids and e-h droplets, and thus prevent the formation of e-h Cooper pairs. ${ }^{48,57,58}$

The fourth reason for choosing $\mathrm{ZnO}$ is that it is possible to create high e-h densities in the bulk of a $\mathrm{ZnO}$ crystal via three-photon absorption of high-intensity infrared laser pulses. Low-temperature luminescence from highly excited $\mathrm{ZnO}$ was studied before in the case of direct excitation by ultraviolet light pulses, ${ }^{59}$ where the short penetration depth limits the excitation layer to a thickness of $50 \mathrm{~nm}$. To avoid emission related to surface impurities and explore the bulk physics, we employed the long penetration depth of infrared light, and excited our crystal slab via three-photon absorption of high-intensity 160-fs 800-nm pulses from an amplified Ti:sapphire laser. An additional advantage of this approach is that spontaneous emission by a preformed e-h Cooper pair triggers stimulated emission from other preformed e-h Cooper pairs as the emitted photon traverses the 500- $\mu \mathrm{m}$-long excited zone. This ASE is relatively easy to measure and recognize. The e-h density in our experiment is controlled via the intensity of the excitation pulse, and calculated from the known three-photon absorption coefficient. ${ }^{53}$

\section{CROSSOVER FROM e-h PLASMA TO PREFORMED e-h COOPER PAIRS}

Measured emission spectra at high e-h density ( $n=9.2 \times$ $10^{24} \mathrm{~m}^{-3}$ ) for decreasing temperature are shown in Fig. 2(a). At $T=50 \mathrm{~K}$ we measure mainly spontaneous emission from an e-h plasma. When the $\mathrm{ZnO}$ crystal is cooled to $4 \mathrm{~K}$, a strong new peak emerges. On the basis of the calculated phase diagram (Fig. 1), it can be expected that at these
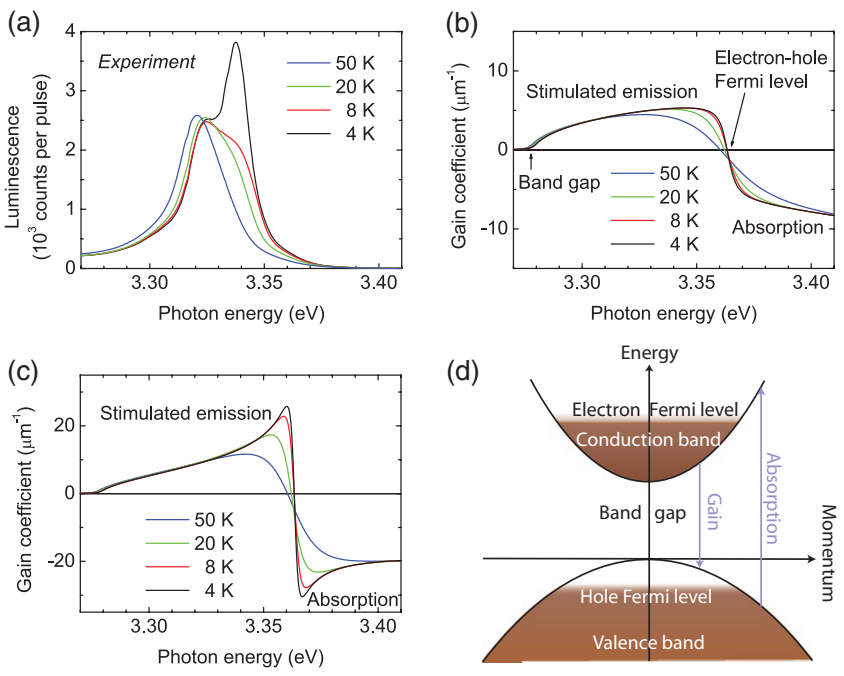

FIG. 2. (Color online) Crossover from the e-h plasma regime to the preformed e-h Cooper-pair regime for decreasing temperature. The e-h density is $n=9.2 \times 10^{24} \mathrm{~m}^{-3}$. (a) Measured emission spectra of the $\mathrm{ZnO}$ crystal. At $T=50 \mathrm{~K}$ there is only emission from an e-h plasma. For decreasing temperature, gain (amplified spontaneous emission) from preformed e-h Cooper pairs appears. (b) Theoretical gain spectra without Coulomb forces between electrons and holes. (c) Theoretical gain spectra taking into account Coulomb forces, showing the appearance of preformed e-h Cooper-pair peaks. (d) Scheme of the band structure and the degenerate e-h gas. For our calculations we used a two-band model including spin degeneracy.

temperatures the e-h gas has entered the preformed e-h Cooper-pair regime.

To find out whether this observed new peak is related to preformed e-h Cooper pairs, we calculated gain spectra of the e-h gas in $\mathrm{ZnO}$, using the many-body theory explained in Ref. 53. This theory has been experimentally verified at room temperature, both for bulk $\mathrm{ZnO}^{53}$ and for $\mathrm{ZnO}$ nanowire lasers. ${ }^{60}$ In this theory, the optical spectra of $\mathrm{ZnO}$ are calculated by solving the statically screened Bethe-Salpeter ladder equation.

The spectra shown in Fig. 2(b) are mean-field spectra, i.e., without the interactions between electrons and holes taken into account. These spectra have been calculated from Eq. (A41) of Ref. 53 with the following low-temperature parameters for the nonrenormalized band gap ${ }^{61}$ and damping: $E_{G, 0}(4 \mathrm{~K})=E_{G, 0}(8 \mathrm{~K})=3.437 \mathrm{eV}, E_{G, 0}(20 \mathrm{~K})=3.436 \mathrm{eV}$, $E_{G, 0}(50 \mathrm{~K})=3.434 \mathrm{eV}$, and $\hbar \gamma_{0}=2.0 \mathrm{meV}$. For the step size $s$ we took $5 \times 10^{6} \mathrm{~m}^{-1}$ instead of $5 \times 10^{7} \mathrm{~m}^{-1}$ to obtain more accurate results. Thus, the matrices to be inverted were $501 \times 501$ instead of $51 \times 51$. All other parameters were the same as for the room-temperature calculation. In all theoretical spectra band-gap renormalization is included, as calculated from the phenomenological formula of Bányai and Koch. ${ }^{52,62}$

In the spectra of Fig. 2(c) the Coulomb forces between electrons and holes have been taken into account. The interaction between the carriers is described by the Yukawa potential, Eq. (1). The spectra have been calculated from Eq. (15) in Ref. 53 with the low-temperature parameters as specified above. Note that the mean-field susceptibility, Eq. (A41), 
can be obtained from the RPA Bethe-Salpeter susceptibility, Eq. (15), by putting $V_{s,\left|\mathbf{k}-\mathbf{k}^{\prime}\right|}=0$, i.e., by ignoring the Coulomb forces between electrons and holes.

Both in Fig. 2(b) and in Fig. 2(c) there is a spectral domain where gain occurs and a domain where absorption prevails, as can be understood from Fig. 2(d). The crucial difference is that for decreasing temperature two peaks appear when Coulomb forces are included: a gain peak just below the e-h Fermi level, and an absorption peak just above this level. In the theory these peaks arise from fluctuations in the e-h pairing fields $\Delta$, directly showing that they are due to (preformed) e-h Cooper pairs. In the two-dimensional case the same physical interpretation of these peaks was obtained in Ref. 42. Importantly, the expectation value of the $\Delta$ fields, i.e., the BCS order parameter, is set to zero in Ref. 53. Therefore, the calculated gain spectra are only valid above $T_{C}$. Note that according to our calculations (Fig. 1) our measurements were performed above $T_{C}$. The influence of the fluctuations in the $\Delta$ fields on the self-energies of the electrons and holes is neglected in this theory. As a result of this, the single-particle density of states does not show pseudogaps in our approximation.

The optical spectra in Ref. 53 were calculated for $\mathbf{E} \perp \mathbf{c}$. In our experiment, the axis of the excited cylinder is parallel to the $c$ axis. ASE therefore mainly has polarization $\mathbf{E} \perp \mathbf{c}$.

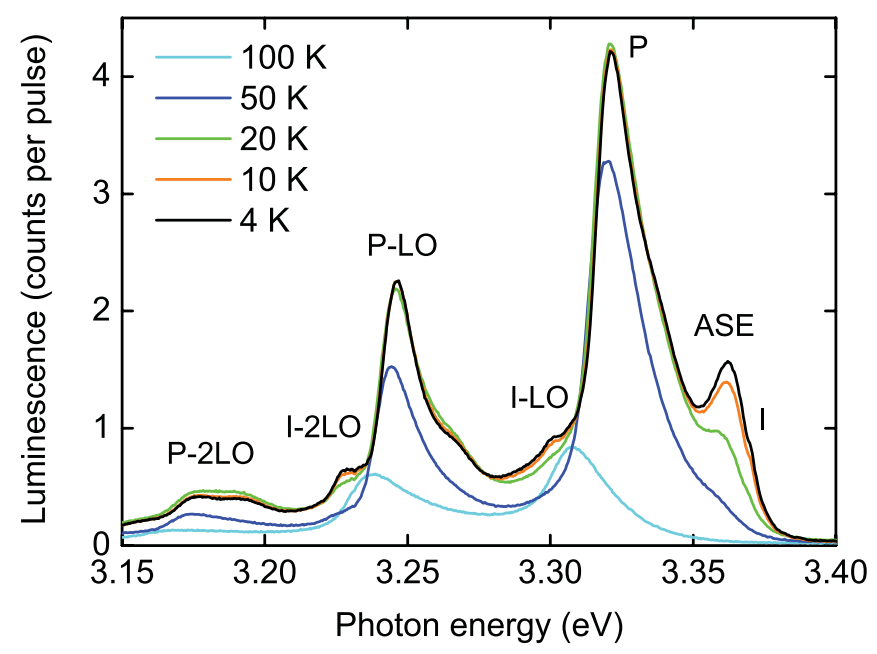

FIG. 3. (Color online) Measured emission spectra from the $\mathrm{ZnO}$ crystal at e-h density $n=1.9 \times 10^{23} \mathrm{~m}^{-3}$ for decreasing temperature, showing the crossover from the e-h plasma to the preformed Cooperpair regime. The $\mathrm{P}$ emission, indicated by $\mathrm{P}$, and the spontaneous emission from excitons bound to aluminum impurities, indicated by I, have first and second phonon replica: Spontaneous emission causing the $\mathrm{P}$ and I peaks also occurs under simultaneous emission of one or two longitudinal-optical (LO) phonons, leading to emission peaks at lower photon energies. These LO phonons have an energy of $72 \mathrm{meV}$. The ASE peak appearing for decreasing temperature is caused by spontaneous emission from e-h pairs in the crossover from excitons to preformed Cooper pairs, amplified by stimulated emission from such pairs. This ASE peak does not have phonon replica, demonstrating that it is not just spontaneous emission like the other peaks. The general shift to higher photon energies for lower temperatures reflects the temperature dependence of the band gap (Ref. 61).
Hence, the expressions given in Ref. 53 can also be used in the present case.

As explained in the Appendix, we used a two-band model, which is a good approximation at low temperatures and densities below about $10^{25} \mathrm{~m}^{-3}$. At higher densities, occupancy of states in the $B$ valence band, and ultimately also in the $C$ valence band, would reduce preformed Cooper-pair gain and shift the gain maximum to lower energies.

Comparison between theory and experimental data in Fig. 2 suggests that the observed new peak is due to stimulated emission from preformed e-h Cooper pairs. Indeed, additional experimental results (Fig. 3) at $n=1.9 \times 10^{23} \mathrm{~m}^{-3}$ show that all emission peaks have longitudinal-optical phonon replica, except the new peak, which is evidence that the new peak is not ordinary spontaneous emission like the other peaks, but is associated with gain. We can therefore attribute this peak to ASE from preformed e-h Cooper pairs. The difference in spectral position between the measured new peak of Fig. 2(a) and the calculated peak of Fig. 2(c) originates from limited precision in the calculation of the band-gap renormalization.

\section{CROSSOVER FROM EXCITONS TO PREFORMED e-h COOPER PAIRS}

In Fig. 4 we explore the crossover from the exciton regime to the preformed Cooper-pair regime at $T=4 \mathrm{~K}$ by tuning the e-h density. At the lowest density the well-known spontaneous emission spectrum is observed: The highest peak is the so-called $\mathrm{P}$ peak, resulting from inelastic scattering of two excitons, where one recombines and the other ionizes. ${ }^{64}$ The emission at $3.371 \mathrm{eV}$ results from recombination of excitons bound to aluminum impurities. ${ }^{65}$ This emission spectrum shows that the e-h gas is in the exciton regime, as could be expected from the calculated phase diagram (Fig. 1). When the density is increased to $7 \times 10^{22} \mathrm{~m}^{-3}$, a remarkable new peak appears, next to the impurity peak. For increasing density this peak grows very fast, even faster than the $\mathrm{P}$ peak, and the spectral distance between the $\mathrm{P}$ peak and this new peak decreases. Comparable emission spectra have been reported in Ref. 59, but without a clear interpretation, leaving these results inconclusive.

To examine the origin of this new peak, we calculated the gain spectra at $T=4 \mathrm{~K}$, shown in Fig. 4(c), using the same theory as for Fig. 2(c). At low densities, these spectra show excitonic absorption and no gain, in agreement with our interpretation of the observed emission as spontaneous emission from an exciton gas. For increasing e-h density, the spectra gradually evolve into a double-peak structure, characteristic for (preformed) e-h Cooper pairs. ${ }^{42}$ This evolution is in agreement with the phase diagram (Fig. 1). Gain appears in the theoretical spectra at $8 \times 10^{22} \mathrm{~m}^{-3}$, very close to the threshold for the new peak. We conclude that the measured new peak originates from ASE from preformed e-h Cooper pairs, or, for densities not far above the Mott density, from bound e-h pairs in the crossover between excitons and preformed e-h Cooper pairs. Note that the impurity emission is still visible, separated from the new peak, showing that these contributions to the emission spectrum have different origins. Interestingly, Mahan excitons in cold doped quantum wells give rise to similar emission features. ${ }^{8}$ 

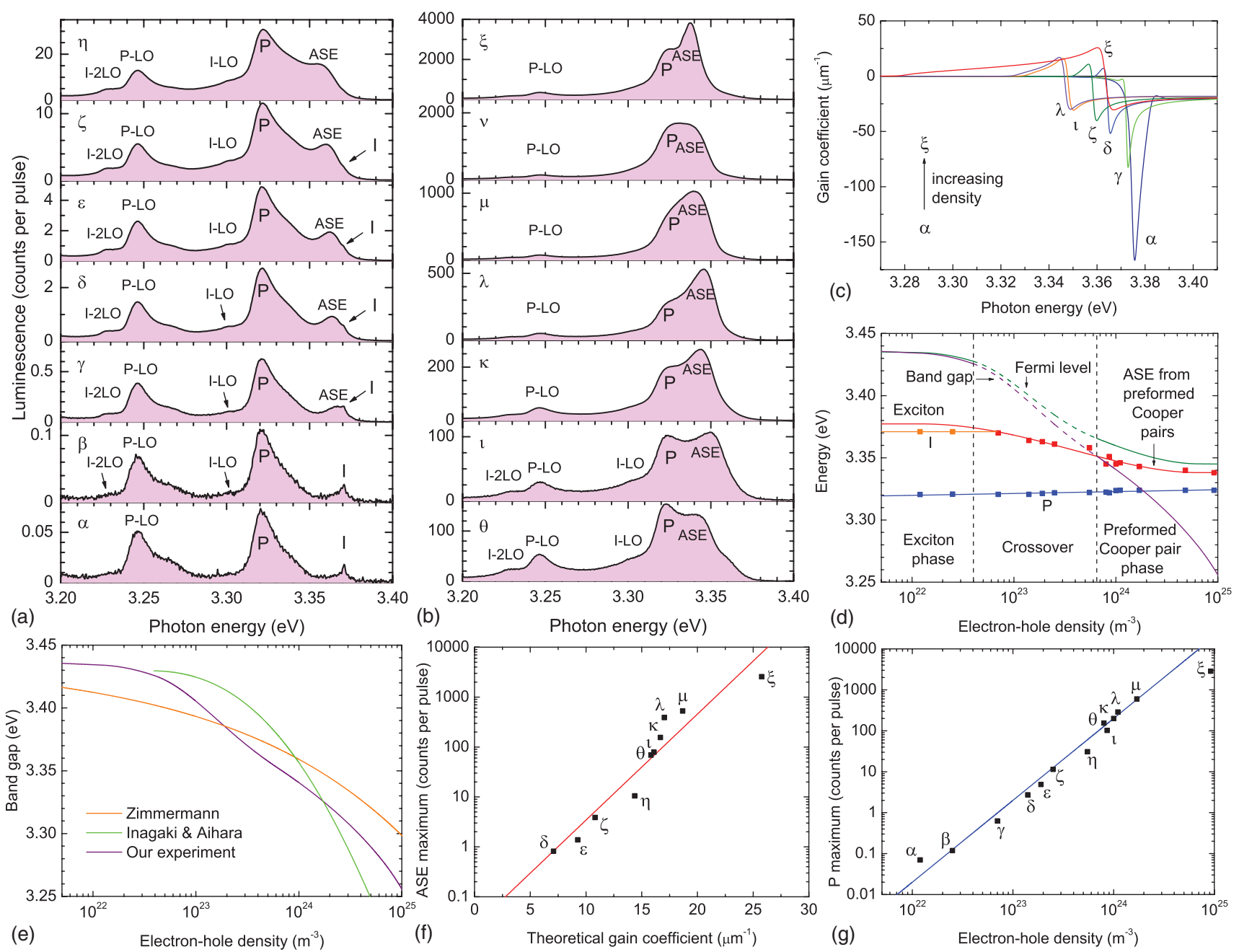

FIG. 4. (Color online) Crossover from the exciton regime to the preformed Cooper-pair regime for increasing e-h density. $T=4 \mathrm{~K}$. (a,b) Measured emission spectra. Greek symbols indicate e-h densities (in $\mathrm{m}^{-3}$ ): $\alpha, 1.2 \times 10^{22} ; \beta, 2.5 \times 10^{22} ; \gamma, 7.0 \times 10^{22} ; \delta, 1.4 \times 10^{23}$; $\varepsilon, 1.9 \times 10^{23} ; \zeta, 2.5 \times 10^{23} ; \eta, 5.5 \times 10^{23} ; \theta, 8.0 \times 10^{23} ; \iota, 8.6 \times 10^{23} ; \kappa, 1.0 \times 10^{24} ; \lambda, 1.1 \times 10^{24} ; \mu, 1.7 \times 10^{24} ; \nu, 4.8 \times 10^{24} ;$ and $\xi$, $9.2 \times 10^{24}$. Indicated are the ASE from preformed e-h Cooper pairs, or from e-h pairs in the crossover from excitons to preformed e-h Cooper pairs, the $\mathrm{P}$ emission $(\mathrm{P})$, the emission from excitons bound to aluminum impurities (I), the first longitudinal optical phonon replica of the $\mathrm{P}$ peak (P-LO), and the first (I-LO) and second (I-2LO) phonon replica of the I peak. (c) Theoretical gain spectra showing the appearance of a preformed e-h Cooper-pair gain peak. Note that the $\mathrm{P}$ peak is absent here, since the theory does not incorporate pair-pair collisions. (d) Squares indicate measured emission energies. The band-gap renormalization and the e-h Fermi level were derived from these. We have drawn dashed lines in the crossover, since a theory is presently lacking for this regime. The distance between the P energy level and the ASE energy level reflects the binding energy of the e-h pairs. (e) Band-gap renormalization. Band gap versus e-h density at $T=4 \mathrm{~K}$ as derived from our experimental results, compared with two curves from the literature: Zimmermann, ${ }^{63}$ calculated at $T=4 \mathrm{~K}$ and Inagaki and Aihara, ${ }^{23}$ calculated at $T=0$. (f) Measured ASE maxima versus theoretical gain coefficient maxima, showing an exponential relation. (g) Measured $\mathrm{P}$ peak maxima versus e-h density, showing a quadratic dependence.

Apparently, the $\mathrm{P}$ peak persists even at high e-h densities where excitons cannot exist. In the preformed Cooper-pair regime we interpret the $\mathrm{P}$ peak as due to inelastic scattering of two preformed e-h Cooper pairs, in which one pair recombines and the other breaks up. This interpretation is in line with calculations by Inagaki and Aihara in the BEC-BCS crossover. ${ }^{23}$ Consequently, the energy separation between the $\mathrm{P}$ maximum and the ASE maximum directly measures the binding energy of the preformed e-h Cooper pairs.

The spectral positions of the measured peaks are analyzed in Fig. 4(d). Here we have indicated the exciton region and the preformed Cooper-pair region, connected by a crossover region above $n=4 \times 10^{22} \mathrm{~m}^{-3}$, the calculated Mott density at $T=4 \mathrm{~K}$. In the crossover, the exciton energy level gradually develops into the preformed e-h Cooper-pair level, which is equal to the spectral position of the gain peak. The diminishing separation between the P and the ASE peak indicates the reduction of the e-h-pair binding energy by screening.

It is important to realize that band-gap renormalization takes place: The band gap narrows for increasing density due to exchange and correlation effects. From the binding energies determined from the measured P-ASE peak separation we 
derive the values of the density-dependent band gap and the e-h Fermi level in the following way. In the exciton regime the band gap is of course located one exciton binding energy above the exciton energy level. In the preformed Cooper-pair regime we do not have such direct information about the value of the band gap. However, we expect a pseudogap around the e-h Fermi level equal to the preformed Cooper-pair binding energy. Therefore, in this regime the Fermi level lies only half of the binding energy above the preformed Cooper-pair level. Ideal gas chemical potentials yield the separation between the band gap and the Fermi level. Based on this argumentation we can draw the band gap and the Fermi level in both the exciton region and in the preformed Cooper-pair region. Unfortunately, for the crossover a theory is presently lacking. Therefore we have drawn dashed lines in this regime. The rather good agreement between the determined band-gap renormalization and curves from the literature [Fig. 4(e)] confirms the correctness of our theory and our interpretation of the experimental data.

In Fig. 4(f) measured ASE maxima are plotted against the theoretical maximum gain coefficients at the e-h densities realized in the experiment. We find an exponential relation, what is to be expected for ASE. The P-peak height depends quadratically on the carrier density [Fig. 4(g)]. This result supports our interpretation of the $\mathrm{P}$ peak as the result of scattering of two excitons or two preformed e-h Cooper pairs.

\section{CONCLUSIONS AND OUTLOOK}

We have observed preformed electron-hole Cooper pairs in a highly excited $\mathrm{ZnO}$ single crystal. Amplified spontaneous emission from preformed e-h Cooper pairs appears as a new peak in the photoluminescence spectra under conditions of high three-photon excitation of the bulk crystal at cryogenic temperatures. Temperature- and density-dependent measurements have revealed the crossover between the e-h plasma regime and the preformed e-h Cooper-pair regime and the crossover between the exciton regime and the preformed e-h Cooper-pair regime. The spectral positions and amplitudes of the peaks were analyzed, and compared with many-body theoretical calculations, confirming our interpretation of the data.

Our calculations of the critical temperature in Fig. 1 indicate that also the superfluid BCS state should be experimentally accessible. It is still an open question how this state could be detected. By using a microcavity setup, Bose-Einstein condensation of two-dimensional e-h Cooper-pair polaritons may occur at even higher temperatures. We hope that our observation of preformed e-h Cooper pairs in a semiconductor will contribute to the understanding of high-temperature superconductivity, exciton-polariton condensates, and excitonic condensation in e-h bilayers and bilayer quantum Hall systems.

\section{ACKNOWLEDGMENTS}

We thank C. R. de Kok and P. Jurrius for technical support, J. M. Vogels, E. E. van Faassen, D. van Oosten, P. van der Straten, and J. Lipfert for discussions and reviewing of our manuscript, and D. van Oosten for drawing the insets of Fig. 1.

\section{APPENDIX}

In this Appendix the computation is explained of the superfluid critical temperature $T_{C}$ of the e-h gas in $\mathrm{ZnO}$ as a function of e-h density in the BCS regime. Below this temperature the e-h gas forms a BCS superfluid, i.e., a condensate of e-h Cooper pairs.

We calculated $T_{C}$ in mean-field approximation by solving the linearized BCS gap equation ${ }^{30,66}$

$$
\begin{aligned}
\Delta_{\mathbf{k}}(n, T)= & \int \frac{d \mathbf{k}^{\prime}}{(2 \pi)^{3}} V_{s,\left|\mathbf{k}-\mathbf{k}^{\prime}\right|}(n, T) \\
& \times \frac{1-f_{\mathbf{k}^{\prime}, e}(n, T)-f_{\mathbf{k}^{\prime}, h}(n, T)}{\mu_{e}(n, T)-\varepsilon_{\mathbf{k}^{\prime}, e}+\mu_{h}(n, T)-\varepsilon_{\mathbf{k}^{\prime}, h}} \Delta_{\mathbf{k}^{\prime}}(n, T),
\end{aligned}
$$

where $\Delta_{\mathbf{k}}(n, T)$ is the momentum-dependent BCS order parameter or gap, and

$$
V_{s,\left|\mathbf{k}-\mathbf{k}^{\prime}\right|}(n, T)=\frac{-e^{2}}{\varepsilon_{0} \varepsilon_{r}\left[\lambda_{s}^{-2}(n, T)+\left|\mathbf{k}-\mathbf{k}^{\prime}\right|^{2}\right]}
$$

is the Yukawa potential in momentum space, i.e., the Fourier transform of Eq. (1). Furthermore, $\varepsilon_{\mathbf{k}, i}$ are the single-particle energies $\varepsilon_{\mathbf{k}, i}=\varepsilon_{k, i}=\hbar^{2} k^{2} /\left(2 m_{i}\right)$, where $i$ stands for $e$, electron, or $h$, hole, and $k=|\mathbf{k}|$. Note that we use a momentumdependent interaction, not a point interaction or separable pseudopotential as in standard BCS theory. Finally,

$$
f_{\mathbf{k}, i}(n, T)=\frac{1}{e^{\left[\varepsilon_{\mathbf{k}, i}-\mu_{i}(n, T)\right] /\left(k_{B} T\right)}+1}
$$

are the Fermi-Dirac distributions of the electron and hole gases.

Equation (A1) is valid for $T \geqslant T_{C}$. For $T>T_{C}$ the BCS gap equation has only one solution, namely, $\Delta_{\mathbf{k}}=0$. This solution corresponds to the normal phase. Below $T_{C}$ also a solution $\Delta_{\mathbf{k}} \neq 0$ is possible, corresponding to the superfluid phase. Since the normal to BCS phase transition is a second-order phase transition in the balanced case, the order parameter $\Delta_{\mathbf{k}}$ continuously increases from zero for $T>T_{C}$ to its maximum at $T=0$. Precisely at $T_{C}$ the order parameter becomes nonzero.

In order to find the highest temperature at which Eq. (A1) has a nonzero solution, we use the fact that for an $s$-wave order parameter we can replace $V_{s,\left|\mathbf{k}-\mathbf{k}^{\prime}\right|}$ by its angle average, depending only on the lengths $k$ and $k^{\prime}$ as

$$
\begin{aligned}
\bar{V}_{s, k, k^{\prime}} & =\frac{1}{2} \int_{0}^{\pi} d \theta \sin \theta V_{s,\left|\mathbf{k}-\mathbf{k}^{\prime}\right|} \\
& =\frac{-e^{2}}{4 \varepsilon_{0} \varepsilon_{r} k k^{\prime}} \ln \left[\frac{\left(k^{2}+k^{\prime 2}+2 k k^{\prime}\right) \lambda_{s}^{2}+1}{\left(k^{2}+k^{\prime 2}-2 k k^{\prime}\right) \lambda_{s}^{2}+1}\right] .
\end{aligned}
$$

In this way, the gap equation is written in the form of a homogeneous Fredholm integral equation of the second kind. Such equations are generally not analytically solvable. In order to obtain a numerical solution, we replace the integral by a finite sum. The discretization is performed following Simpson's rule and leads to the simple eigenvalue problem

$$
\vec{\Delta}(n, T)=\overrightarrow{\vec{U}}(n, T) \cdot \vec{\Delta}(n, T) .
$$




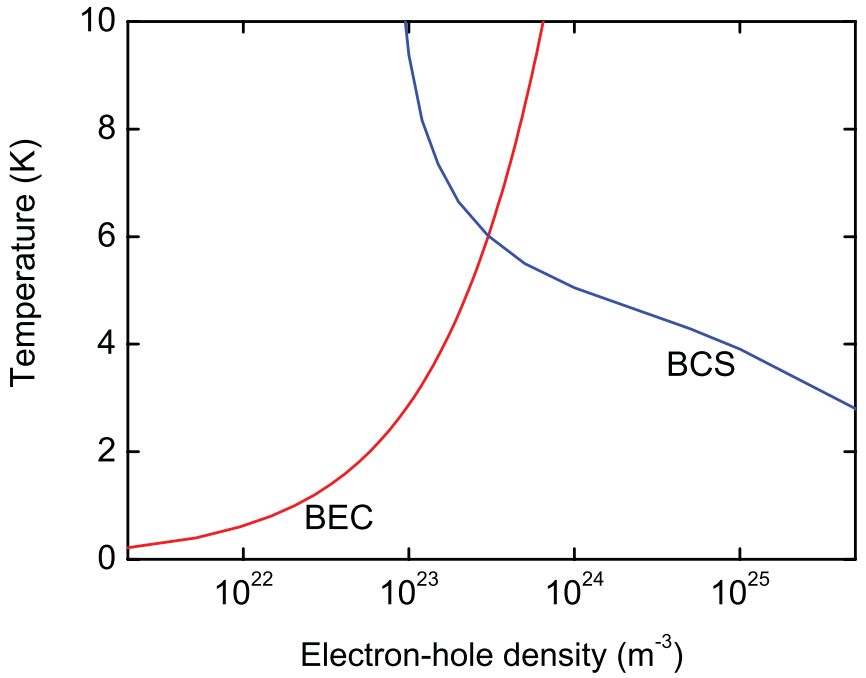

FIG. 5. (Color online) Critical temperature. $T_{C}$ calculated in the BEC regime [Eq. (2)] and $T_{C}$ calculated in the BCS regime by solving the BCS equation [Eq. (A1)].

The gap has become a vector $\vec{\Delta}(n, T)$ with length $p$, where $p$ is the number of steps in the discretization. The quantity $\overrightarrow{\vec{U}}(n, T)$ is a $p \times p$ matrix, of which the elements are given by

$U_{i j}(n, T)=\frac{w_{j} k_{j}^{2} \bar{V}_{s, k_{i}, k_{j}}(n, T)\left[1-f_{k_{j}, e}(n, T)-f_{k_{j}, h}(n, T)\right]}{2 \pi^{2}\left[\mu_{e}(n, T)-\varepsilon_{k_{j}, e}+\mu_{h}(n, T)-\varepsilon_{k_{j}, h}\right]}$,

where $w_{j}$ are the Simpson weights for each discretization point. For each density $n$ we approach $T_{C}$ from above and search for a nontrivial solution of Eq. (A5). The critical temperature $T_{C}$ is the temperature at which $\vec{U}(n, T)$ has eigenvalue 1 . The results for the critical temperature in the BEC and in the BCS regime are shown in Figs. 1 and 5.

For all calculations in this paper we used an isotropic parabolic two-band model, including spin degeneracy. $\mathrm{ZnO}$ has one twofold spin-degenerate conduction band and three twofold spin-degenerate valence bands, called $A, B$, and $C$. The three valence bands are split as a result of spin-orbit coupling and the hexagonal crystal field. The $A B$ splitting equals $10 \mathrm{meV}$, and the $A C$ splitting $44 \mathrm{meV} .{ }^{67}$ In our model only the conduction band and the the highest valence band, the $A$ band, are taken into account.

At low temperatures and densities where the hole Fermi energy is smaller than the $A B$ splitting this is a good approximation, since almost all holes are in the $A$ band. According to the hole masses determined by Hümmer ${ }^{56}$ the hole Fermi energy equals $10 \mathrm{meV}$ at $n=2 \times 10^{24} \mathrm{~m}^{-3}$. According to the nonisotropic energy-dependent hole masses calculated by Lambrecht et al. ${ }^{67}$ however, this is only the case at $n=8 \times 10^{24} \mathrm{~m}^{-3}$. For the calculations of the Mott density and of the critical temperature in the BEC regime, inclusion of the $B$ and $C$ bands in the calculation would not make a difference. For the critical temperature in the BCS regime an effect would show up at high densities where the occupancy in the $B$ band is sufficiently high that it significantly affects the hole Fermi level. When that happens, a distinction has to be made between the BCS order parameter related to correlations between the $A$ band and the conduction band and the BCS order parameter related to correlations between the $B$ band and the conduction band. Because of the presence of density imbalance, the physics changes and exotic phases such as the Sarma phases and the FFLO phase could possibly show up. *j.i.dijkhuis@uu.nl

${ }^{1}$ J. Bardeen, L. N. Cooper, and J. R. Schrieffer, Phys. Rev. 108, 1175 (1957).

${ }^{2}$ C. A. Regal, M. Greiner, and D. S. Jin, Phys. Rev. Lett. 92, 040403 (2004).

${ }^{3}$ C. Chin, M. Bartenstein, A. Altmeyer, S. Riedl, S. Jochim, J. Hecker Denschlag, and R. Grimm, Science 305, 1128 (2004).

${ }^{4}$ M. W. Zwierlein, J. R. Abo-Shaeer, A. Schirotzek, C. H. Schunck, and W. Ketterle, Nature (London) 435, 1047 (2005).

${ }^{5}$ L. V. Keldysh and Yu. V. Kopaev, Fiz. Tverd. Tela (Leningrad) 6, 2791 (1964) [Sov. Phys. Solid State 6, 2219 (1965)].

${ }^{6}$ C. Comte and P. Nozières, J. Physique 43, 1069 (1982).

${ }^{7}$ G. D. Mahan, Phys. Rev. 153, 882 (1967).

${ }^{8}$ M. S. Skolnick, J. M. Rorison, K. J. Nash, D. J. Mowbray, P. R. Tapster, S. J. Bass, and A. D. Pitt, Phys. Rev. Lett. 58, 2130 (1987).

${ }^{9}$ T. Makino, C. H. Chia, K. Tamura, Y. Segawa, M. Kawasaki, A. Ohtomo, and H. Koinuma, Phys. Rev. B 65, 121201 (2002).

${ }^{10}$ M. Feneberg, J. Däubler, K. Thonke, R. Sauer, P. Schley, and R. Goldhahn, Phys. Rev. B 77, 245207 (2008).

${ }^{11}$ S. Shokhovets, K. Köhler, O. Ambacher, and G. Gobsch, Phys. Rev. B 79, 045201 (2009).
${ }^{12}$ A. Schleife, C. Rödl, F. Fuchs, K. Hannewald, and F. Bechstedt, Phys. Rev. Lett. 107, 236405 (2011).

${ }^{13}$ J. E. Baarsma, J. Armaitis, R. A. Duine, and H. T. C. Stoof, Phys. Rev. A 85, 033631 (2012).

${ }^{14}$ J. des Cloizeaux, J. Phys. Chem. Solids 26, 259 (1965).

${ }^{15}$ D. Jérome, T. M. Rice, and W. Kohn, Phys. Rev. 158, 462 (1967).

${ }^{16}$ F. J. Di Salvo, D. E. Moncton, and J. V. Waszczak, Phys. Rev. B 14, 4321 (1976).

${ }^{17}$ C. Monney, C. Battaglia, H. Cercellier, P. Aebi, and H. Beck, Phys. Rev. Lett. 106, 106404 (2011).

${ }^{18}$ B. Bucher, P. Steiner, and P. Wachter, Phys. Rev. Lett. 67, 2717 (1991).

${ }^{19}$ Y. Wakisaka, T. Sudayama, K. Takubo, T. Mizokawa, M. Arita, H. Namatame, M. Taniguchi, N. Katayama, M. Nohara, and H. Takagi, Phys. Rev. Lett. 103, 026402 (2009).

${ }^{20} \mathrm{P}$. Nozières and C. Comte, J. Physique 43, 1083 (1982).

${ }^{21}$ C. Comte and G. Mahler, Phys. Rev. B 34, 7164 (1986).

${ }^{22}$ T. Iida, Y. Hasegawa, H. Higashimura, and M. Aihara, Phys. Rev. B 47, 9328 (1993).

${ }^{23}$ T. J. Inagaki and M. Aihara, Phys. Rev. B 65, 205204 (2002).

${ }^{24}$ P. B. Littlewood, P. R. Eastham, J. M. J. Keeling, F. M. Marchetti, B. D. Simons, and M. H. Szymanska, J. Phys.: Condens. Matter 16, S3597 (2004). 
${ }^{25}$ K. Mizoo, T. J. Inagaki, Y. Ueshima, and M. Aihara, J. Phys. Soc. Jpn. 74, 1745 (2005).

${ }^{26}$ D. Kremp, D. Semkat, and K. Henneberger, Phys. Rev. B 78, 125315 (2008).

${ }^{27}$ T. Ogawa and Y. Tomio, J. Lumin. 129, 1478 (2009).

${ }^{28}$ K. Yoshioka, E. Chae, and M. Kuwata-Gonokami, Nat. Commun. 2, 328 (2011).

${ }^{29}$ X. Zhu, P. B. Littlewood, M. S. Hybertsen, and T. M. Rice, Phys. Rev. Lett. 74, 1633 (1995).

${ }^{30}$ S. Conti, G. Vignale, and A. H. MacDonald, Phys. Rev. B 57, R6846 (1998).

${ }^{31}$ P. Pieri, D. Neilson, and G. C. Strinati, Phys. Rev. B 75, 113301 (2007).

${ }^{32}$ A. A. High, J. R. Leonard, A. T. Hammack, M. M. Fogler, L. V. Butov, A. V. Kavokin, K. L. Campman, and A. C. Gossard, Nature (London) 483, 584 (2012).

${ }^{33}$ A. A. High, J. R. Leonard, M. Remeika, L. V. Butov, M. Hanson, and A. C. Gossard, Nano Lett., doi: 10.1021/n1300983n (2012).

${ }^{34}$ M. Kellogg, J. P. Eisenstein, L. N. Pfeiffer, and K. W. West, Phys. Rev. Lett. 93, 036801 (2004).

${ }^{35}$ E. Tutuc, M. Shayegan, and D. A. Huse, Phys. Rev. Lett. 93, 036802 (2004).

${ }^{36}$ Yu. E. Lozovik and A. A. Sokolik, JETP Lett. 87, 55 (2008).

${ }^{37}$ B. Seradjeh, J. E. Moore, and M. Franz, Phys. Rev. Lett. 103, 066402 (2009).

${ }^{38}$ D. Tilahun, B. Lee, E. M. Hankiewicz, and A. H. MacDonald, Phys. Rev. Lett. 107, 246401 (2011).

${ }^{39}$ J. Kasprzak, M. Richard, S. Kundermann, A. Baas, J. Jeambrun, J. M. J. Keeling, F. M. Marchetti, M. H. Szymańska, R. André, J. L. Staehli, V. Savona, P. B. Littlewood, B. Deveaud, and Le Si Dang, Nature (London) 443, 409 (2006).

${ }^{40}$ R. Balili, V. Hartwell, D. Snoke, L. Pfeiffer, and K. West, Science 316, 1007 (2007).

${ }^{41}$ N. Y. Kim, K. Kusudo, C. Wu, N. Masumoto, A. Löffler, S. Höfling, N. Kumada, L. Worschech, A. Forchel, and Y. Yamamoto, Nat. Phys. 7, 681 (2011).

${ }^{42}$ S. Schmitt-Rink, C. Ell, and H. Haug, Phys. Rev. B 33, 1183 (1986).

${ }^{43}$ B. Zenker, D. Ihle, F. X. Bronold, and H. Fehske, Phys. Rev. B 85 , 121102 (2012).

${ }^{44}$ H. B. Yang, J. D. Rameau, P. D. Johnson, T. Valla, A. Tsvelik, and G. D. Gu, Nature (London) 456, 77 (2008).
${ }^{45}$ T. Kondo, Y. Hamaya, A. D. Palszewski, T. Takeuchi, J. S. Wen, Z. J. Xu, G. Gu, J. Schmalian, and A. Kaminski, Nat. Phys. 7, 21 (2011).

${ }^{46}$ J. P. Gaebler, J. T. Stewart, T. E. Drake, D. S. Jin, A. Perali, P. Pieri, and G. C. Strinati, Nat. Phys. 6, 569 (2010).

${ }^{47}$ J. M. Blatt, K. W. Böer, and W. Brandt, Phys. Rev. 126, 1691 (1962).

${ }^{48}$ S. A. Moskalenko and D. W. Snoke, Bose-Einstein Condensation of Excitons and Biexcitons (Cambridge University Press, Cambridge, 2000).

${ }^{49}$ E. Tiesinga, B. J. Verhaar, and H. T. C. Stoof, Phys. Rev. A 47, 4114 (1993).

${ }^{50}$ C. A. Regal, C. Ticknor, J. L. Bohn, and D. S. Jin, Nature (London) 424, 47 (2003).

${ }^{51}$ S. Jochim, M. Bartenstein, A. Altmeyer, G. Hendl, S. Riedl, C. Chin, J. Hecker Denschlag, and R. Grimm, Science 302, 2101 (2003).

${ }^{52} \mathrm{H}$. Haug and S. W. Koch, Quantum Theory of the Optical and Electronic Properties of Semiconductors, 4th ed. (World Scientific, Singapore, 2004).

${ }^{53}$ M. A. M. Versteegh, T. Kuis, H. T. C. Stoof, and J. I. Dijkhuis, Phys. Rev. B 84, 035207 (2011).

${ }^{54}$ C. Kittel and H. Kroemer, Thermal Physics, 2nd ed. (Freeman, New York, 1980).

${ }^{55}$ K. J. Button, D. R. Cohn, M. van Ortenbert, B. Lax, E. Mollwo, and R. Helbig, Phys. Rev. Lett. 28, 1637 (1972).

${ }^{56}$ K. Hümmer, Phys. Status Solidi B 56, 249 (1973).

${ }^{57}$ W. F. Brinkman and T. M. Rice, Phys. Rev. B 7, 1508 (1973).

${ }^{58}$ G. A. Thomas, T. G. Phillips, T. M. Rice, and J. C. Hensel, Phys. Rev. Lett. 31, 386 (1973).

${ }^{59}$ H. Priller, J. Brückner, Th. Gruber, C. Klingshirn, H. Kalt, A. Waag, H. J. Ko, and T. Yao, Phys. Status Solidi B 241, 587 (2004).

${ }^{60}$ M. A. M. Versteegh, D. Vanmaekelbergh, and J. I. Dijkhuis, Phys. Rev. Lett. 108, 157402 (2012).

${ }^{61}$ R. Hauschild, H. Priller, M. Decker, J. Brückner, H. Kalt, and C. Klingshirn, Phys. Status Solidi C 3, 976 (2006).

${ }^{62}$ L. Bányai and S. W. Koch, Z. Phys. B 63, 283 (1986).

${ }^{63}$ R. Zimmermann, Phys. Status Solidi B 146, 371 (1988).

${ }^{64}$ J. M. Hvam, Solid State Commun. 12, 95 (1973).

${ }^{65}$ B. K. Meyer, J. Sann, S. Lautenschläger, M. R. Wagner, and A. Hoffmann, Phys. Rev. B 76, 184120 (2007).

${ }^{66}$ H. T. C. Stoof, K. B. Gubbels, and D. B. M. Dickerscheid, Ultracold Quantum Fields (Springer, Dordrecht, 2009).

${ }^{67}$ W. R. L. Lambrecht, A. V. Rodina, S. Limpijumnong, B. Segall, and B. K. Meyer, Phys. Rev. B 65, 075207 (2002). 